\title{
Genetic diversity reduction in improved durum wheat cultivars of Morocco as revealed by microsatellite markers
}

\author{
Fatima Henkrar ${ }^{1,2,3,4}$, Jamal El-Haddoury ${ }^{3}$, Hassan Ouabbou ${ }^{3}$, Nasserlehaq Nsarellah ${ }^{3}$, Driss Iraqi², Najib Bendaou ${ }^{4}$, Sripada Mahabala
} Udupa $^{1 *}$

\author{
IInternational Center for Agricultural Research in the Dry Areas, \\ P0 Box 6299, Rue Hafiane Cherkaoui - Rabat - Morocco. \\ ${ }^{2}$ National Institute for Agricultural Research, PO Box 415, Av. \\ Ennasr - Rabat - Morocco. \\ ${ }^{3}$ National Institute for Agricultural Research, PO Box 589 - \\ Settat - Morocco. \\ ${ }^{4}$ Mohammed V University-Agdal/Faculty of Sciences - Plant \\ Physiology and Biotechnology Laboratory, PO Box 1014 - \\ Rabat - Morocco. \\ *Corresponding author <s.udupa@cgiar.org>
}

Edited by: Leonardo Oliveira Medici

Received February 20, 2015

Accepted June 17, 2015
ABSTRACT: It has been argued that genetic diversity in crop varieties has been on the decline in recent times due to plant breeding. This can have serious consequences for both the genetic vulnerability of crops and their plasticity when responding to changes in production environments. It is, therefore, vital for plant breeding programs to maintain sufficient diversity in the cultivars deployed for multi-period cultivation. In this study, to understand the temporal genetic diversity in durum wheat, 21 improved durum wheat cultivars released in Morocco, since 1956 and five exotic cultivars currently used in crossing programs were analyzed using 13 microsatellite markers. The analysis revealed a total of 44 alleles and average genetic diversity of 0.485 with genetic distances ranging from 0.077 to 0.846 at 13 microsatellite loci in Moroccan durum wheat cultivars. All the durum cultivars of Morocco could be distinguished using the 13 microsatellite markers. The total number of alleles and unique alleles were highest in cultivars developed before 1990, decreasing in cultivars developed during the 1990s and 2000s, indicating that recent durum breeding efforts have reduced allelic richness in recent cultivars. Thus, deployment of exotic durum wheat lines in breeding programs could enhance genetic diversity in durum wheat cultivars.

Keywords: Triticum turgidum L. subsp. durum, cultivar characterization, SSR markers

\section{Introduction}

Durum wheat (Triticum turgidum L. subsp. durum, $2 \mathrm{n}=4 \mathrm{x}=28 ; \mathrm{AABB}$ ) is a tetraploid wheat, mainly grown in the Mediterranean basin, and other parts of the world for human consumption. Morocco produces around 1.2 million tons of durum wheat annually. However, most years Morocco imports durum wheat to supply its growing demand. Therefore, the improvement of such a crop in terms of yield and quality is necessary. The overall objective of Moroccan wheat breeding was and remains the development of durum wheat genotypes with high genetic potential for yield and quality. Efforts to improve durum wheat were initiated in 1921, through mass-selection and introduction of exotic cultivars. Variability was generated through hybridization between better performing local and exotic cultivars (Jlibene and Nsarellah, 2011). In all these periods, the introduced and improved varieties had a great impact on Moroccan wheat production.

Genetic variation in registered varieties is fundamental to the improvement of future breeding programs by providing a basis for selection of superior parental combinations and predictions of progeny performance (Haile et al., 2013). Analyses of genetic divergence and estimation of genetic distance between parents are useful for choosing parents in wheat hybridization programs (Islam, 2004). The loss of variation in crops due to the modernization of agriculture has been described as genetic erosion. Genetic erosion of cultivated diversity is reflected in a modernization bottleneck at diversity levels that occurred during the history of the crop (Wouw et al., 2009). It is crucial to formulate an idea about genetic diversity changes in existing gene-pools of cultivated crops in order to understand the impact of plant breeding on crop genetic diversity (Fu et al., 2005) and it could make crop improvement more efficient by the direct accumulation of desired alleles.

Molecular markers play a pivotal role in varietal evaluation; it can speed up the process and decrease the amount of plant material that needs to be screened in such experiments (Astarini et al., 2004). Microsatellite markers have been used for analysis of genetic diversity and identification of indigenous landraces and modern cultivars (Khanjari et al., 2007; Wang et al., 2007) and also used for temporal variation in wheat (Roussel et al., 2004 and 2005; Fu et al., 2006; Figliuolo et al., 2007; Huang et al., 2007; $\mathrm{Fu}$ and Somers, 2009). This study analyzed the use of microsatellite markers for cultivar genetic diversity analysis, genetic distance estimation and to understand temporal changes in genetic diversity and allele richness in Moroccan durum wheat cultivars developed since 1956.

\section{Materials and Methods}

\section{Plant materials}

A total of 26 durum wheat cultivars consisting of 21 improved cultivars released for cultivation in Morocco (Nsarellah et al., 2005) were provided by the National Gene Bank of Morocco, INRA, Settat, Morocco (Table 1). Five potential exotic cultivars which are important as donors in the breeding program, namely Vitron (Spain), Strong Field (Canada), Medora (Canada), Sceptre (Canada) and UC1113-GPC-B1 (USA) were also included in this study. 
Table 1 - Improved durum wheat cultivars of Morocco procured from the National Gene Bank of Moroccoa.

\begin{tabular}{lcl}
\hline Cultivar & Registration year & \multicolumn{1}{c}{ Pedigree } \\
\hline Kyperonda & 1956 & Selection from 'Cyprus' population \\
Cocorit & 1975 & Rae/4Tc60//STW63/3/AA'S' \\
Selbera & 1982 & Old landrace \\
Marzak & 1984 & Selection from CIMMYTs 'PYTII' \\
Karim & 1985 & Bittern 'S' same as JO'S'.AA':S'//FG'S' \\
Sebou & 1987 & Selection from 'Grebe'S' population. \\
Bel Bachir & 1988 & Improved cultivar introduced from Tunisia \\
Tensift & 1988 & Njoro 226 selection from unknown Cyprus population. \\
Oum Rabia & 1988 & Selection from 'Cyprus3' population. \\
Sarif & 1988 & Selection from the Lds/Mut//Teal'S' population \\
Jawhar & 1993 & Moroccan selection in hybridization with introduced material \\
Ourgh & 1995 & Moroccan crossing and selection of introduced material \\
Tarek & 1995 & Selection from a population derived from Moroccan line and introduced lines \\
Amjad & 1995 & Moroccan crossing and selection on introduced material \\
Merjana & 1996 & Selection from a population derived from crossing Moroccan line and introduced lines \\
Tomouh & 1997 & Selection on introduced material in Morocco \\
Marouane & 2003 & Crossing and Moroccan selection \\
Nassira & 2003 & Crossing and Moroccan selection \\
Amria & 2003 & Crossing and Moroccan selection \\
Irden & 2003 & Crossing and Moroccan selection \\
Icamor & 2006 & Crossing of parents from Morocco and ICARDA and selection at Morocco and ICARDA \\
\hline aKindly provided by Hassan Ouabbou & &
\end{tabular}

\section{DNA extraction and microsatellites analysis}

Genomic DNA was extracted from 4-week-old seedlings ( $5 \mathrm{~cm}$ of young leaf blades) of individual cultivars using the CTAB (cetyltrimethylammonium bromide) method of Saghai-Maroof et al., (1984) with minor modifications (Udupa et al., 1999): use of $2 \% \mathrm{CTAB}$ buffer for extraction instead of $1 \%$ CTAB and use of sterile distilled water for dissolution of the final DNA pellet instead of $10 \mathrm{mM}$ NH4OAc/0.25 mM EDTA (ethylenediaminetetraacetic acid). Quality and quantity of the isolated DNA were assessed by intactness and intensity of the DNA band, respectively, obtained after electrophoresis of $3 \mu \mathrm{L}$ of the isolated DNA in $1 \%$ agarose (w/v) gel, stained with ethidium bromide and visualized under Ultra Violet (U.V.) rays. The intensity of the band of isolated DNA was compared to known concentrations of lambda DNA digested with EcoRI and HindIII restriction enzymes.

Thirteen polymorphic microsatellites (Table 2) were used in this study. The Polymerase Chain Reactions (PCRs) were performed in total volume of $10 \mu \mathrm{L}$, containing $1 \mathrm{x}$ PCR buffer $\left(1.5 \mathrm{mM} \mathrm{MgCl}_{2}\right), 200 \mu \mathrm{M}$ of each dNTPs (deoxyribose nucleotide triphosphates), 10 pmoles of each primer, 0.5 U of Taq DNA polymerase and approximately $50 \mathrm{ng}$ of genomic DNA. The amplification reaction was generated in the Eppendorf Master cycler with initial denaturation for five minutes at 94 ${ }^{\circ} \mathrm{C}$, followed by 35 cycles of each cycle with 30 seconds denaturation at $94{ }^{\circ} \mathrm{C}, 30$ seconds annealing at $59{ }^{\circ} \mathrm{C}$, 45 seconds extension at $72{ }^{\circ} \mathrm{C}$. Final extension was carried out at $72{ }^{\circ} \mathrm{C}$ for five minutes followed by cooling at $4^{\circ} \mathrm{C}$ for an undefined period. Amplified products were separated on $6 \%(\mathrm{w} / \mathrm{v})$ denaturing polyacrylamide gels. The amplified bands were detected by silver staining. The size of each band was estimated simultaneously by means of a 100-bp DNA Ladder.

\section{Data analysis}

PowerMarker software (Ver. 3.0; Liu and Muse, 2005) was used to calculate genetic diversity, number of alleles and the shared allele genetic distance (Jin and Chakraborty, 1993). The average number of alleles, unique alleles and genetic diversity for each temporal group were calculated. These temporal groups were also compared with the exotic varieties currently used in breeding programs. To determine genetic divergence, genetic distances were calculated for each pair of temporal groups. A dendrogram was constructed based on genetic distance by using the Neighbor-joining (NJ) method (Saitou and Nei, 1987) and visualized using MEGA5 software (Tamura et al., 2011). An Analysis of Molecular Variance Analysis (AMOVA) and Principal Coordinates Analysis (PCoA) were undertaken using GenAlEx 6.5 software (Peakall and Smouse, 2012). The FPtest (Fu, 2010) was performed (with 50,000 random permutations) to test the significance of differences in allelic count between the temporal groups.

\section{Results}

\section{Microsatellite polymorphism}

A total of 44 alleles were detected for the Moroccan cultivars and 25 alleles for the 5 exotic cultivars 
(Table 3). The number of alleles per locus ranged from 2 (Xbarc263, Xwmc89, Xpsp2999 and Xwmc24) to 6 (for Xgwm577) with an average number of 3.38. The $13 \mathrm{mi}-$ crosatellites used were sufficient to differentiate all the 21 Moroccan cultivars and the five exotic cultivars. Average genetic diversity $(H)$ calculated for all markers and Moroccan genotypes was 0.485 (Table 3). The Xgwm136, Xgwm577 and Xgwm389 markers were the most informative and showed the highest value $(0.671,0.626$ and 0.621 , respectively).
The 21 Moroccan durum wheat cultivars were discriminated using 13 microsatellite markers (Figure 1). The genetic distance (Table 4) was lowest between Amria and Irden, Marjana and Amjad, Sebou and Kyperonda, Bel Bachir and Vitron, Sarif and Vitron, and Tarek and Vitron (0.077), indicating that these accessions are closely related to each other. The highest genetic distance was observed between Karim and Kyperonda, Ourgh and Kyperonda, Ourgh and Sebou, and Marouane and Selbera (0.846). Cluster analysis based on the

Table 2 - Locus name, sequences, repeat motif of 13 microsatellite markers used in this study.

\begin{tabular}{lccccc}
\hline Locus & Location & Forward primer (5'-3') & Reverse primer (5'-3') & Repeat motif* & Reference \\
\hline Xgwm33 & 1A & GGAGTCACACTTGTTTGTGCA & CACTGCACACCTAACTACCTGC & (GA)19 & Röder et al., 1998 \\
Xgwm389 & 3B & ATC ATG TCG ATC TCC TTG ACG & TGC CAT GCA CAT TAG CAG AT & (CT)14(GT)16 & Röder et al., 1998 \\
Xgwm146 & 7B & CCA AAA AAA CTG CCT GCA TG & CTC TGG CAT TGC TCC TTG G & (GA)5GG(GA)20 & Röder et al., 1998 \\
Xgwm397 & 4A & TGT CAT GGA TTA TTT GGT CGG & CTG CAC TCT CGG TAT ACC AGC & (CT)21 & Röder et al., 1998 \\
Xgwm136 & 1A & GAC AGC ACC TTG CCC TTT G & CAT CGG CAA CAT GCT CAT C & (CT)58 & Röder et al., 1998 \\
Xbarc263 & 1A & GGAAGGCGTCAGCACTAGGCAAC & GGCTTCTAGGTGCTGCGGCTTTTGTC & (ATT)17 & Ward et al., 2003 \\
Xgwm130 & 7A 2B 7B & AGC TCT GCT TCA CGA GGA AG & CTC CTC TTT ATA TCG CGT CCC & (GT)22 & Röder et al., 1998 \\
Xwmc89 & 4A & ATGTCCACGTGCTAGGGAGGTA & TTGCCTCCCAAGACGAAATAAC & (CA)19 or (CT)8 & Somers et al., 2004 \\
Xgwm193 & 6B & CTT TGT GCA CCT CTC TCT CC & AAT TGT GTT GAT GAT TTG GGG & (CT)24imp(CA)8 & Röder et al., 1998 \\
Xgwm273 & 1B & ATT GGA CGG ACA GAT GCT TT & AGC AGT GAG GAA GGG GAT C & (GA)18 & Röder et al., 1998 \\
Xpsp2999 & 1A & TCCCGCCATGAGTCAATC & TTGGGAGACACATTGGCC & (CAG)n(CAA)n & Devos et al.., 1995 \\
Xwmc24 & 1A & GTGAGCAATTTTGATTATACTG & TACCCTGATGCTGTAATATGTG & (GT)28 & Somers et al., 2004 \\
Xgwm577 & 7B & ATG GCA TAA TTT GGT GAA ATT G & TGT TTC AAG CCC AAC TTC TAT T & (CA)14(TA)6 & Röder et al., 1998 \\
\hline
\end{tabular}

${ }^{*}$ Repeat motif in bread wheat var. Chinese Spring

Table 3 - Changes in the number of alleles, unique alleles and genetic diversity over periods in durum wheat cultivars of Morocco and their comparison to the exotic cultivars.

\begin{tabular}{|c|c|c|c|c|c|c|c|c|c|c|c|c|c|c|c|c|c|c|c|c|}
\hline \multirow[b]{2}{*}{ Locus } & \multirow[b]{2}{*}{ 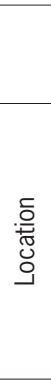 } & \multicolumn{4}{|c|}{$\begin{array}{l}\text { Moroccan cultivars } \\
\text { before 1990s }\end{array}$} & \multicolumn{4}{|c|}{$\begin{array}{l}\text { Moroccan cultivars of } \\
\text { 1990s }\end{array}$} & \multicolumn{4}{|c|}{$\begin{array}{l}\text { Moroccan cultivars of } \\
2000 \mathrm{~s}\end{array}$} & \multicolumn{3}{|c|}{$\begin{array}{c}\text { Moroccan cultivars } \\
\text { before 1990s, } \\
1990 \text { s and 2000s }\end{array}$} & \multicolumn{4}{|c|}{$\begin{array}{l}\text { Exotic } \\
\text { cultivars }\end{array}$} \\
\hline & & 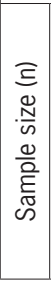 & 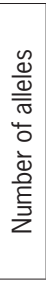 & 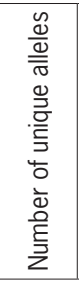 & 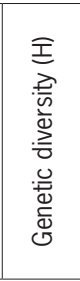 & 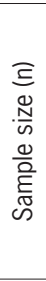 & 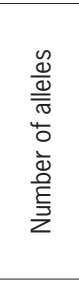 & 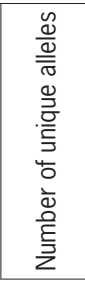 & 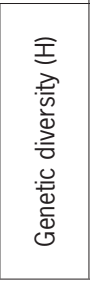 & 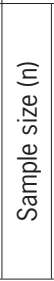 & 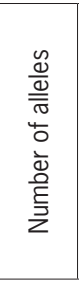 & 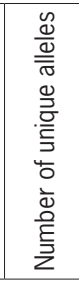 & 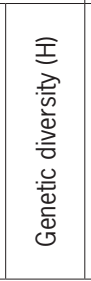 & 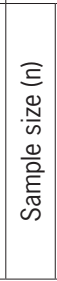 & 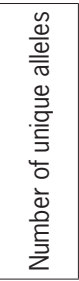 & 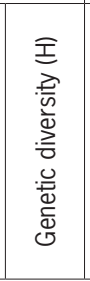 & 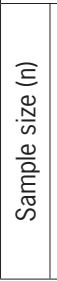 & 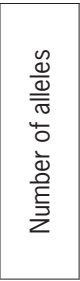 & 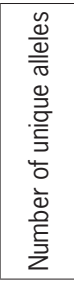 & 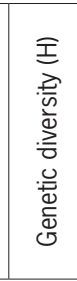 \\
\hline Xgwm33 & $1 \mathrm{~A}$ & 10 & 3 & 1 & 0.460 & 6 & 1 & 0 & 0 & 5 & 3 & 1 & 0.640 & 21 & 4 & 0.463 & 5 & 1 & 0 & 0 \\
\hline Xgwm389 & $3 B$ & 10 & 4 & 2 & 0.580 & 6 & 2 & 0 & 0.444 & 5 & 2 & 0 & 0.320 & 21 & 4 & 0.621 & 5 & 3 & 1 & 0.560 \\
\hline Xgwm146 & $7 B$ & 10 & 4 & 3 & 0.640 & 6 & 1 & 0 & 0 & 5 & 2 & 1 & 0.320 & 21 & 5 & 0.463 & 5 & 1 & 0 & 0 \\
\hline Xgwm397 & $4 \mathrm{~A}$ & 10 & 3 & 1 & 0.580 & 6 & 2 & 0 & 0.500 & 5 & 2 & 0 & 0.480 & 21 & 3 & 0.544 & 5 & 2 & 0 & 0.480 \\
\hline Xgwm136 & $1 \mathrm{~A}$ & 10 & 4 & 1 & 0.640 & 6 & 2 & 0 & 0.500 & 5 & 2 & 0 & 0.480 & 21 & 4 & 0.671 & 5 & 2 & 1 & 0.320 \\
\hline Xbarc263 & $1 \mathrm{~A}$ & 10 & 2 & 1 & 0.180 & 6 & 1 & 0 & 0 & 5 & 1 & 0 & 0 & 21 & 2 & 0.091 & 5 & 1 & 0 & 0 \\
\hline Xgwm130 & $\begin{array}{l}7 A \\
2 B \\
7 B\end{array}$ & 10 & 3 & 1 & 0.460 & 6 & 2 & 0 & 0.444 & 5 & 3 & 1 & 0.640 & 21 & 4 & 0.531 & 5 & 2 & 0 & 0.320 \\
\hline Xwmc89 & $4 \mathrm{~A}$ & 10 & 2 & 0 & 0.480 & 6 & 2 & 0 & 0.500 & 5 & 1 & 0 & 0 & 21 & 2 & 0.490 & 5 & 1 & 0 & 0 \\
\hline Xgwm193 & $6 B$ & 10 & 3 & 1 & 0.540 & 6 & 2 & 0 & 0.500 & 5 & 2 & 0 & 0.480 & 21 & 3 & 0.526 & 5 & 3 & 1 & 0.560 \\
\hline Xgwm273 & $1 \mathrm{~B}$ & 10 & 2 & 0 & 0.420 & 6 & 3 & 1 & 0.611 & 5 & 2 & 0 & 0.480 & 21 & 3 & 0.503 & 5 & 2 & 0 & 0.320 \\
\hline Xpsp2999 & $1 \mathrm{~A}$ & 10 & 2 & 0 & 0.420 & 6 & 1 & 0 & 0 & 5 & 2 & 0 & 0.480 & 21 & 2 & 0.408 & 5 & 2 & 0 & 0.320 \\
\hline Xwmc24 & $1 \mathrm{~A}$ & 10 & 2 & 0 & 0.480 & 6 & 2 & 0 & 0.278 & 5 & 1 & 0 & 0 & 21 & 2 & 0.363 & 5 & 2 & 0 & 0.480 \\
\hline Xgwm577 & $7 B$ & 10 & 5 & 3 & 0.720 & 6 & 2 & 1 & 0.278 & 5 & 2 & 0 & 0.480 & 21 & 6 & 0.626 & 5 & 3 & 1 & 0.640 \\
\hline Total & & & 39 & 14 & & & 23 & 2 & & & 25 & 3 & & & 44 & & & 25 & 4 & \\
\hline Mean & & & 3 & 1.077 & 0.508 & & 1.769 & 0.154 & 0.312 & & 1.923 & 0.231 & 0.369 & & 3.384 & 0.485 & & 1.923 & 0.308 & 0.308 \\
\hline Standard deviation $( \pm)$ & & & 1 & 1.038 & 0.136 & & 0.599 & 0.376 & 0.234 & & 0.641 & 0.439 & 0.230 & & 1.261 & 0.147 & & 0.760 & 0.480 & 0.237 \\
\hline
\end{tabular}


Henkrar et al.

\begin{tabular}{|c|c|c|c|c|c|c|c|c|c|c|c|c|c|c|c|c|c|c|c|c|c|c|c|c|c|c|}
\hline uod!! & $\begin{array}{l}\infty \\
\stackrel{\infty}{\sim} \\
0 \\
0\end{array}$ & $\begin{array}{l}n \\
0 \\
0 \\
0\end{array}$ & 今. & \begin{tabular}{l}
$\tilde{W}$ \\
\multirow{\sigma}{*}{} \\
0
\end{tabular} & \begin{tabular}{l} 
ô \\
\multirow{\sigma}{0}{} \\
0
\end{tabular} & $\begin{array}{l}\infty \\
\stackrel{\infty}{\rho} \\
0 \\
0\end{array}$ & 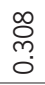 & \begin{tabular}{l} 
Ð \\
\multirow{0}{*}{} \\
0
\end{tabular} & \begin{tabular}{|l}
1 \\
6 \\
0 \\
0
\end{tabular} & $\begin{array}{l}\infty \\
\stackrel{n}{n} \\
0\end{array}$ & 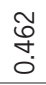 & 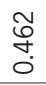 & \begin{tabular}{l}
$\tilde{W}$ \\
\multirow{\sigma}{*}{} \\
0
\end{tabular} & 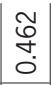 & $\begin{array}{l}\infty \\
\tilde{n} \\
\tilde{n} \\
0\end{array}$ & $\begin{array}{l}\infty \\
\text { లె. } \\
0\end{array}$ & $\begin{array}{l}\hat{a} \\
\hat{O} \\
0\end{array}$ & $\begin{array}{l}\text { Оิ } \\
\text { Oे }\end{array}$ & $\begin{array}{l}\qquad \\
\overrightarrow{0} \\
0 \\
0\end{array}$ & $\mid \begin{array}{l}1 \\
0 \\
0 \\
0\end{array}$ & 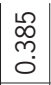 & 命 & $\begin{array}{l}\overrightarrow{\widetilde{N}} \\
\stackrel{0}{0}\end{array}$ & 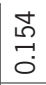 & 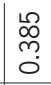 & \& \\
\hline 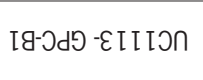 & $\begin{array}{l}\stackrel{1}{0} \\
\text { m } \\
0 \\
0\end{array}$ & $\begin{array}{l}\infty \\
\\
\\
0\end{array}$ & $\begin{array}{l}\tilde{\sigma} \\
0 \\
0\end{array}$ & $\begin{array}{l}\stackrel{2}{0} \\
\infty \\
m \\
0\end{array}$ & $\begin{array}{l}\tilde{o} \\
\text { ơ } \\
0\end{array}$ & \begin{tabular}{|l|}
$n$ \\
6 \\
0 \\
0
\end{tabular} & $\stackrel{\vec{\sim}}{\sim}$ & $\begin{array}{l}\stackrel{+}{5} \\
\stackrel{0}{0}\end{array}$ & $\begin{array}{l}9 \\
0 \\
0 \\
0\end{array}$ & $\begin{array}{l}\infty \\
\\
\\
0\end{array}$ & 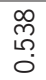 & $\begin{array}{l}\infty \\
\text { లె } \\
0\end{array}$ & $\begin{array}{l}\infty \\
\text { లె } \\
0\end{array}$ & $\begin{array}{l}\infty \\
\text { ల్ } \\
0\end{array}$ & 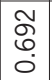 & 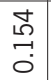 & \begin{tabular}{|c|} 
\\
$\infty$ \\
$m$ \\
0 \\
0
\end{tabular} & $\begin{array}{l}\tilde{U} \\
\dot{W} \\
0\end{array}$ & $\begin{array}{l}9 \\
\stackrel{2}{0} \\
\dot{0}\end{array}$ & $\begin{array}{l}\infty \\
\tilde{N} \\
\stackrel{0}{0} \\
0\end{array}$ & 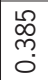 & $\begin{array}{l}\tilde{O} \\
\mathscr{6} \\
0\end{array}$ & $\begin{array}{l}\tilde{U} \\
\dot{y} \\
0\end{array}$ & $\begin{array}{l}\tilde{O} \\
0 \\
0\end{array}$ & $\begin{array}{l}8 \\
8 \\
0\end{array}$ & \\
\hline unowol & $\begin{array}{l}\text { To } \\
\text { q. }\end{array}$ & \begin{tabular}{|l|} 
\\
6 \\
0 \\
0
\end{tabular} & $\overrightarrow{\tilde{n}}$ & $\begin{array}{l}\infty \\
\tilde{n} \\
\stackrel{0}{0}\end{array}$ & $\begin{array}{l}\widetilde{W} \\
\text { qu } \\
0\end{array}$ & $\begin{array}{l}\infty \\
\prod_{0}^{\infty} \\
0 \\
0\end{array}$ & $\begin{array}{l}\stackrel{0}{\infty} \\
\text { m. } \\
0\end{array}$ & $\begin{array}{l}\infty \\
\stackrel{n}{n} \\
\stackrel{n}{0}\end{array}$ & $\begin{array}{l}\infty \\
\tilde{n} \\
\stackrel{0}{0} \\
0\end{array}$ & $\begin{array}{l}n \\
6 \\
0 \\
0\end{array}$ & 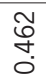 & $\begin{array}{l}\infty \\
\stackrel{n}{n} \\
\stackrel{n}{0}\end{array}$ & 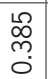 & 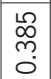 & $\begin{array}{l}\tilde{U} \\
\mathscr{f} \\
0\end{array}$ & $\begin{array}{l}\tilde{U} \\
\mathscr{f} \\
0\end{array}$ & $\begin{array}{l}\vec{n} \\
\stackrel{0}{0}\end{array}$ & 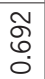 & \begin{tabular}{|l|} 
\\
0 \\
6 \\
0 \\
0
\end{tabular} & $\begin{array}{l}\infty \\
\tilde{n} \\
\stackrel{0}{0}\end{array}$ & $\begin{array}{l}\text { To } \\
\text { f+. } \\
0\end{array}$ & $\stackrel{\vec{m}}{N}$ & 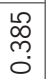 & ৪ & & \\
\hline \#!SUә & $\begin{array}{l}\text { ơ } \\
\text { : }\end{array}$ & $\begin{array}{l}\text { శ్ } \\
0 \\
0\end{array}$ & $\begin{array}{l}\infty \\
\text { Oొ } \\
0\end{array}$ & $\begin{array}{l}\infty \\
\stackrel{\infty}{n} \\
\stackrel{0}{0}\end{array}$ & $\begin{array}{l}\infty \\
\stackrel{\infty}{n} \\
0 \\
0\end{array}$ & $\begin{array}{l}10 \\
0 \\
0 \\
0\end{array}$ & $\begin{array}{l}\infty \\
\stackrel{\infty}{\sim} \\
\stackrel{0}{0}\end{array}$ & 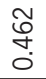 & 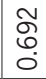 & $\begin{array}{l}\text { O్ } \\
0 \\
0\end{array}$ & $\begin{array}{l}\infty \\
\stackrel{\infty}{n} \\
0 \\
0\end{array}$ & $\begin{array}{l}\infty \\
\stackrel{\infty}{\Omega} \\
\stackrel{0}{0}\end{array}$ & 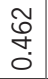 & $\begin{array}{l}\infty \\
\stackrel{\infty}{\Omega} \\
\stackrel{0}{0}\end{array}$ & 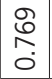 & $\begin{array}{l}\infty \\
\text { లె } \\
0\end{array}$ & $\begin{array}{l}\overrightarrow{\tilde{N}} \\
\tilde{0}\end{array}$ & $\begin{array}{l}\qquad \\
0 \\
0 \\
0\end{array}$ & 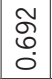 & $\begin{array}{l}\infty \\
\tilde{N} \\
\stackrel{0}{0} \\
0\end{array}$ & $\begin{array}{l}\infty \\
\stackrel{\infty}{0} \\
\stackrel{0}{0}\end{array}$ & $\begin{array}{l}0 \\
0 \\
\text { r. } \\
0\end{array}$ & $\begin{array}{l}8 \\
\text { ¿ } \\
\circ\end{array}$ & & & \\
\hline уәде & $\begin{array}{l}0 \\
0 \\
0 \\
0\end{array}$ & $\begin{array}{l}\tilde{\delta} \\
0 \\
0\end{array}$ & 苫 & $\begin{array}{l}\infty \\
\sim \\
\stackrel{1}{0} \\
0\end{array}$ & 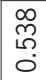 & \begin{tabular}{|l|} 
\\
6 \\
0 \\
0
\end{tabular} & $\begin{array}{l}\stackrel{\infty}{\infty} \\
\\
0\end{array}$ & 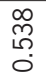 & $\begin{array}{l}\infty \\
\sim \\
\stackrel{0}{0} \\
0\end{array}$ & $\begin{array}{l}\Omega \\
6 \\
0 \\
0\end{array}$ & $\begin{array}{l}\infty \\
0 \\
1 \\
0 \\
0\end{array}$ & 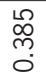 & $\begin{array}{l}\infty \\
\tilde{N} \\
\stackrel{\infty}{0} \\
0\end{array}$ & \begin{tabular}{|l|}
$\infty$ \\
0 \\
1 \\
0 \\
0 \\
0
\end{tabular} & $\begin{array}{l}\tilde{U} \\
\dot{\sigma} \\
0 \\
0\end{array}$ & $\begin{array}{l}\stackrel{L}{\infty} \\
\text { m } \\
0 \\
0\end{array}$ & $\begin{array}{l}0 \\
0 \\
-1 \\
0\end{array}$ & $\begin{array}{l}\Omega \\
0 \\
0 \\
0\end{array}$ & \begin{tabular}{|l|} 
\\
0 \\
$\sim$ \\
0 \\
0 \\
\end{tabular} & $\begin{array}{l}\infty \\
\tilde{N} \\
\\
0\end{array}$ & $\begin{array}{l}\infty \\
\text { Oे } \\
\text { m. }\end{array}$ & $\begin{array}{l}8 \\
8 \\
0\end{array}$ & & & & \\
\hline рәәцภిиоגక & $\begin{array}{l}0 \\
\tilde{\sigma} \\
0 \\
0\end{array}$ & $\begin{array}{l}0 \\
6 \\
0 \\
0 \\
\end{array}$ & 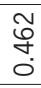 & 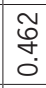 & $\begin{array}{l}0 \\
\overrightarrow{6} \\
0 \\
0\end{array}$ & $\begin{array}{l}0 \\
0 \\
0 \\
0 \\
\end{array}$ & 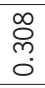 & \begin{tabular}{l}
$\widetilde{v}$ \\
\multirow{6}{*}{} \\
0
\end{tabular} & $\begin{array}{l}\infty \\
\text { Dִ } \\
0 \\
0\end{array}$ & 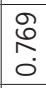 & 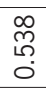 & $\begin{array}{l}\text { 㕝 } \\
\stackrel{7}{0}\end{array}$ & $\begin{array}{l}\qquad \\
\overrightarrow{6} \\
\ddot{0}\end{array}$ & 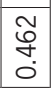 & $\begin{array}{l}\infty \\
\stackrel{\infty}{\sim} \\
\stackrel{0}{0}\end{array}$ & 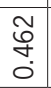 & 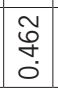 & $\begin{array}{l}\stackrel{L}{\infty} \\
\text { m } \\
0\end{array}$ & \begin{tabular}{|l|}
$\infty$ \\
$\tilde{N}$ \\
$\tilde{\omega}$ \\
0
\end{tabular} & $\begin{array}{l}\infty \\
\text { ñ } \\
\text { مُ } \\
0\end{array}$ & $\begin{array}{l}8 \\
80 \\
0\end{array}$ & & & & & \\
\hline еגәવ|әS & $\begin{array}{l}\infty \\
\infty \\
m \\
0 \\
0\end{array}$ & శ్ & $\begin{array}{l}0 \\
6 \\
0 \\
0\end{array}$ & $\begin{array}{l}\qquad \\
6 \\
0 \\
0\end{array}$ & 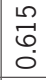 & $\begin{array}{l}\tilde{\sigma} \\
0 \\
0 \\
0\end{array}$ & 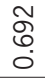 & 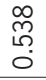 & \begin{tabular}{l}
$\tilde{J}$ \\
\multirow{\sigma}{0}{} \\
0
\end{tabular} & $\begin{array}{l}0 \\
\infty \\
\infty \\
0 \\
0\end{array}$ & $\begin{array}{l}n \\
6 \\
0 \\
0\end{array}$ & \begin{tabular}{l} 
ర్t \\
\multirow{0}{*}{}
\end{tabular} & $\begin{array}{l}\stackrel{L}{\infty} \\
\text { m. } \\
0\end{array}$ & 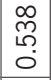 & 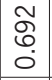 & $\begin{array}{l}\infty \\
\stackrel{N}{\Omega} \\
\stackrel{0}{0}\end{array}$ & \begin{tabular}{|l|}
2 \\
0 \\
0 \\
0
\end{tabular} & \begin{tabular}{l} 
ర్ \\
\multirow{0}{0}{} \\
0
\end{tabular} & $\begin{array}{l}\infty \\
\tilde{n} \\
\stackrel{\rho}{0} \\
0.0\end{array}$ & $\begin{array}{l}8 \\
0 \\
0\end{array}$ & & & & & & \\
\hline noqəS & $\begin{array}{l}0 \\
\stackrel{0}{0} \\
0\end{array}$ & 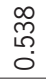 & $\begin{array}{l}\stackrel{0}{0} \\
0 \\
0\end{array}$ & $\begin{array}{l}0 \\
0 \\
0 \\
0\end{array}$ & $\begin{array}{l}0 \\
\$ \\
\infty \\
0\end{array}$ & $\begin{array}{l}\infty \\
\stackrel{\infty}{0} \\
\stackrel{0}{0} \\
0\end{array}$ & శ్ & $\begin{array}{l}0 \\
\infty \\
\infty \\
0\end{array}$ & $\begin{array}{l}\hat{O} \\
\text { Oे } \\
0\end{array}$ & 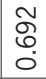 & $\begin{array}{l}2 \\
0 \\
0 \\
0\end{array}$ & $\begin{array}{l}\stackrel{2}{0} \\
\overrightarrow{0} \\
0\end{array}$ & 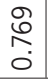 & 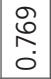 & 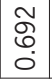 & $\begin{array}{l}0 \\
\infty \\
\infty \\
0\end{array}$ & 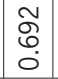 & $\begin{array}{l}\stackrel{1}{0} \\
\overrightarrow{0} \\
0\end{array}$ & 웅 & & & & & & & \\
\hline әд dәכS & 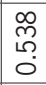 & $\begin{array}{l}\text { م) } \\
\vec{\sigma} \\
0\end{array}$ & $\begin{array}{l}\text { శ్ } \\
\text { م. } \\
0\end{array}$ & $\begin{array}{l}0 \\
\ddot{\sigma} \\
\dot{0}\end{array}$ & $\begin{array}{l}\widetilde{\sigma} \\
\text { ర్ } \\
0\end{array}$ & $\begin{array}{l}10 \\
6 \\
0 \\
0\end{array}$ & $\begin{array}{l}\text { م) } \\
\vec{\omega} \\
\ddot{0}\end{array}$ & 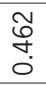 & $\begin{array}{l}\qquad 0 \\
\ddot{\sigma} \\
\dot{0}\end{array}$ & 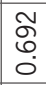 & $\begin{array}{l}\infty \\
\stackrel{\infty}{n} \\
\stackrel{0}{0} \\
0\end{array}$ & $\underset{\sim}{\stackrel{\vec{N}}{\sim}}$ & $\begin{array}{l}\infty \\
\stackrel{N}{0} \\
0 \\
0\end{array}$ & $\begin{array}{l}\infty \\
\stackrel{\infty}{\Omega} \\
0 \\
0\end{array}$ & 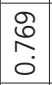 & 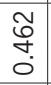 & 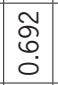 & $\begin{array}{l}\circ \\
8 \\
\circ \\
\circ\end{array}$ & & & & & & & & \\
\hline !!ues & $\begin{array}{l}\infty \\
\sim \\
\\
0 \\
0\end{array}$ & $\begin{array}{l}\text { శ్ } \\
\text { ல }\end{array}$ & $\begin{array}{l}\text { 点 } \\
\text { - } \\
0\end{array}$ & $\begin{array}{l}\infty \\
\sim \\
\stackrel{1}{0} \\
0 \\
0\end{array}$ & $\begin{array}{l}\tilde{o} \\
0 \\
0\end{array}$ & \begin{tabular}{|l|}
0 \\
6 \\
0 \\
0
\end{tabular} & 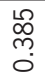 & 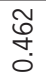 & $\begin{array}{l}\tilde{N} \\
6 \\
0 \\
0\end{array}$ & $\begin{array}{l}0 \\
6 \\
0 \\
0\end{array}$ & $\begin{array}{l}\infty \\
\tilde{N} \\
\\
0\end{array}$ & 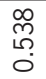 & $\begin{array}{l}\tilde{U} \\
\dot{o} \\
\dot{0}\end{array}$ & 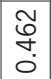 & $\begin{array}{l}\qquad 0 \\
6 \\
0 \\
0\end{array}$ & $\begin{array}{l}\infty \\
\text { ల్ } \\
\text { o. }\end{array}$ & $\begin{array}{l}8 \\
8 \\
0\end{array}$ & & & & & & & & & \\
\hline 48ి.n० & 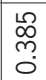 & \begin{tabular}{|l|} 
\\
6 \\
0 \\
0
\end{tabular} & $\begin{array}{l}\stackrel{\leftrightarrow}{\infty} \\
\stackrel{\infty}{m} \\
0\end{array}$ & \begin{tabular}{l}
$\tilde{W}$ \\
\multirow{\sigma}{0}{} \\
0
\end{tabular} & $\begin{array}{l}\infty \\
\text { ల్ } \\
0\end{array}$ & 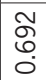 & $\begin{array}{l}\infty \\
\text { లె } \\
0\end{array}$ & 茍 & \begin{tabular}{|l|} 
\\
\\
$\infty$ \\
0 \\
0
\end{tabular} & $\begin{array}{l}\text { ô } \\
\text { o. }\end{array}$ & \begin{tabular}{|l|} 
\\
6 \\
0 \\
0
\end{tabular} & 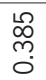 & 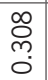 & $\begin{array}{l}\infty \\
\text { లn } \\
0\end{array}$ & $\begin{array}{l}\mathscr{0} \\
\stackrel{0}{0} \\
0\end{array}$ & ৪ & & & & & & & & & & \\
\hline e!qeywno & $\begin{array}{l}L \\
0 \\
0 \\
0\end{array}$ & $\begin{array}{l}\tilde{\sigma} \\
0 \\
0\end{array}$ & $\begin{array}{l}\stackrel{0}{0} \\
\overrightarrow{6} \\
0\end{array}$ & $\begin{array}{l}1 \\
0 \\
0 \\
0\end{array}$ & $\begin{array}{l}\stackrel{1}{0} \\
\stackrel{0}{0} \\
0\end{array}$ & $\begin{array}{l}10 \\
0 \\
0 \\
0\end{array}$ & \begin{tabular}{l} 
ర్t \\
\multirow{0}{0}{}
\end{tabular} & $\begin{array}{l}\stackrel{2}{0} \\
\overrightarrow{0} \\
0\end{array}$ & $\begin{array}{l}0 \\
0 \\
0 \\
0\end{array}$ & $\begin{array}{l}\text { م) } \\
\stackrel{0}{0} \\
0\end{array}$ & $\begin{array}{l}0 \\
0 \\
0 \\
0\end{array}$ & $\begin{array}{l}\stackrel{2}{0} \\
\overrightarrow{0} \\
0\end{array}$ & $\begin{array}{l}\stackrel{\infty}{\sim} \\
\stackrel{\sim}{\rho} \\
0\end{array}$ & 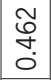 & ○े & & & & & & & & & & & \\
\hline Eג!SSEN & $\underset{\tilde{N}}{\stackrel{\vec{v}}{0}}$ & $\begin{array}{l}\infty \\
0 \\
\sim \\
0 \\
0\end{array}$ & $\begin{array}{l}\infty \\
\stackrel{\infty}{n} \\
\stackrel{0}{0}\end{array}$ & 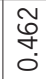 & $\begin{array}{l}\text { ज़ } \\
\stackrel{1}{0}\end{array}$ & \begin{tabular}{|l|} 
\\
6 \\
0 \\
0
\end{tabular} & $\begin{array}{l}\stackrel{+}{0} \\
\stackrel{1}{0}\end{array}$ & $\begin{array}{l}\text { 点 } \\
\stackrel{0}{0}\end{array}$ & $\begin{array}{l}\tilde{~} \\
6 \\
0 \\
0\end{array}$ & $\begin{array}{l}\infty \\
\stackrel{\infty}{0} \\
\stackrel{0}{0}\end{array}$ & $\begin{array}{l}\infty \\
0 \\
\\
0 \\
0\end{array}$ & $\begin{array}{l}\infty \\
\stackrel{\infty}{\Omega} \\
\stackrel{0}{0}\end{array}$ & $\begin{array}{l}\stackrel{+}{0} \\
\overrightarrow{0} \\
0\end{array}$ & $\begin{array}{l}8 \\
8 \\
0\end{array}$ & & & & & & & & & & & & \\
\hline eue!ıəW & $\begin{array}{l}\hat{O} \\
0 \\
0\end{array}$ & 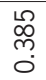 & $\begin{array}{l}\infty \\
\stackrel{\infty}{n} \\
\stackrel{0}{0} \\
0\end{array}$ & $\begin{array}{l}\tilde{O} \\
\tilde{O} \\
0\end{array}$ & $\begin{array}{l}\infty \\
\text { లె } \\
0\end{array}$ & $\begin{array}{l}\tilde{\sigma} \\
\tilde{\sigma} \\
0\end{array}$ & $\begin{array}{l}\infty \\
\text { లె } \\
0\end{array}$ & \begin{tabular}{l}
\multirow{H}{0}{} \\
$\stackrel{0}{0}$ \\
0
\end{tabular} & 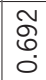 & 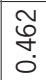 & 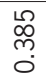 & $\begin{array}{l}\infty \\
\substack{\infty \\
\\
0}\end{array}$ & $\begin{array}{l}8 \\
8 \\
0\end{array}$ & & & & & & & & & & & & & \\
\hline еגорәW & $\begin{array}{l}\infty \\
\stackrel{n}{0} \\
0 \\
0\end{array}$ & \begin{tabular}{|l|} 
\\
6 \\
0 \\
0 \\
0
\end{tabular} & $\begin{array}{l}\infty \\
\stackrel{n}{n} \\
\stackrel{0}{0}\end{array}$ & \begin{tabular}{|l|} 
\\
$\infty$ \\
$m$ \\
0
\end{tabular} & $\begin{array}{l}\text { ه్ } \\
\text { రీ }\end{array}$ & \begin{tabular}{|l|} 
\\
0 \\
6 \\
0 \\
0
\end{tabular} & $\begin{array}{l}\stackrel{0}{\infty} \\
\text { లn } \\
0\end{array}$ & 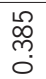 & \begin{tabular}{|l|} 
\\
0 \\
0 \\
0
\end{tabular} & 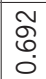 & $\begin{array}{l}\infty \\
\tilde{n} \\
\sim \\
0 \\
0\end{array}$ & $\begin{array}{l}8 \\
\varnothing \\
\circ\end{array}$ & & & & & & & & & & & & & & \\
\hline yeziew & $\begin{array}{l}L_{\infty}^{\infty} \\
\text { m } \\
0\end{array}$ & $\overrightarrow{\widetilde{N}}$ & $\begin{array}{l}\infty \\
\stackrel{\infty}{n} \\
\text { ?ִ } \\
0\end{array}$ & 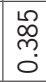 & 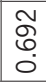 & 苟 & \begin{tabular}{l}
$\widetilde{\mho}$ \\
\multirow{0}{*}{}
\end{tabular} & \begin{tabular}{l}
$\tilde{\sigma}$ \\
\multirow{0}{*}{} \\
0
\end{tabular} & 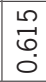 & \begin{tabular}{l}
$\widetilde{\sigma}$ \\
\multirow{\sigma}{*}{} \\
0
\end{tabular} & 8 & & & & & & & & & & & & & & & \\
\hline әuenoגeW & 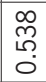 & $\begin{array}{l}\infty \\
\text { Oे } \\
\text { ల. }\end{array}$ & $\begin{array}{l}\qquad 0 \\
\overrightarrow{6} \\
0\end{array}$ & 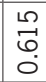 & 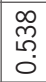 & $\begin{array}{l}\stackrel{1}{\infty} \\
\stackrel{0}{0} \\
0\end{array}$ & $\begin{array}{l}\text { Uै } \\
\stackrel{+}{0} \\
\dot{0}\end{array}$ & \begin{tabular}{l}
$\widetilde{\sigma}$ \\
\multirow{0}{0}{}
\end{tabular} & $\begin{array}{l}0 \\
0 \\
0 \\
0\end{array}$ & 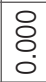 & & & & & & & & & & & & & & & & \\
\hline ерunoגədאY & $\begin{array}{l}\text { శ్ } \\
6 \\
0\end{array}$ & $\begin{array}{l}\qquad 0 \\
6 \\
0 \\
0\end{array}$ & $\begin{array}{l}\qquad 0 \\
6 \\
0 \\
0\end{array}$ & $\begin{array}{l}9 \\
0 \\
0 \\
0\end{array}$ & 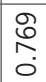 & $\begin{array}{l}\omega \\
6 \\
6 \\
0\end{array}$ & $\begin{array}{l}\text { శ్ } \\
\text { ல் }\end{array}$ & $\begin{array}{l}0 \\
\$ \\
\infty \\
0\end{array}$ & $\begin{array}{l}8 \\
\\
\\
\end{array}$ & & & & & & & & & & & & & & & & & \\
\hline ш!1еу & $\underset{\tilde{N}}{\stackrel{\vec{v}}{0}}$ & $\begin{array}{l}\tilde{\sigma} \\
\tilde{\sigma} \\
0\end{array}$ & $\begin{array}{l}\infty \\
\text { ñ } \\
0 \\
0\end{array}$ & $\begin{array}{l}\infty \\
\text { ల. } \\
\text { ○. }\end{array}$ & $\begin{array}{l}\infty \\
\text { లె } \\
0\end{array}$ & $\begin{array}{l}\infty \\
\prod_{n}^{\infty} \\
0 \\
0\end{array}$ & $\begin{array}{l}\stackrel{+}{\omega} \\
\stackrel{-}{0}\end{array}$ & $\begin{array}{l}8 \\
\text { ¿ }\end{array}$ & & & & & & & & & & & & & & & & & & \\
\hline деумег & 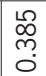 & $\begin{array}{l}\tilde{O} \\
\mathscr{\sigma} \\
0 \\
0\end{array}$ & $\begin{array}{l}\stackrel{\sim}{\infty} \\
\stackrel{m}{m} \\
0\end{array}$ & 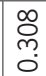 & $\begin{array}{l}\infty \\
\text { ల్ } \\
0\end{array}$ & $\begin{array}{l}\infty \\
\sim \\
\sim \\
0 \\
0\end{array}$ & \begin{tabular}{l}
8 \\
\hdashline
\end{tabular} & & & & & & & & & & & & & & & & & & & \\
\hline иәрд| & \begin{tabular}{l}
$\widetilde{\sigma}$ \\
\multirow{\sigma}{0}{} \\
0
\end{tabular} & $\begin{array}{l}\hat{\sigma} \\
\text { Oे } \\
0\end{array}$ & $\begin{array}{l}\qquad 0 \\
6 \\
0 \\
0\end{array}$ & 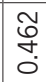 & 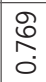 & 8 & & & & & & & & & & & & & & & & & & & & \\
\hline дошеग & $\begin{array}{l}\stackrel{L}{\infty} \\
\text { m } \\
0 \\
0\end{array}$ & $\begin{array}{l}\text { శ్ } \\
6 \\
0\end{array}$ & $\begin{array}{l}\tilde{O} \\
0 \\
0 \\
0\end{array}$ & $\begin{array}{l}\infty \\
\sim \\
\stackrel{1}{0} \\
0\end{array}$ & $\begin{array}{l}8 \\
8 \\
0\end{array}$ & & & & & & & & & & & & & & & & & & & & & \\
\hline $7 ! 10000$ & 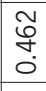 & $\begin{array}{l}\infty \\
\stackrel{\infty}{\infty} \\
\stackrel{0}{0} \\
0\end{array}$ & $\begin{array}{l}\text { đo } \\
\text { ণै } \\
0\end{array}$ & $\begin{array}{l}8 \\
\\
0 \\
\end{array}$ & & & & & & & & & & & & & & & & & & & & & & \\
\hline ג!પગеgાәg & $\begin{array}{l}1 \\
0 \\
0 \\
0\end{array}$ & 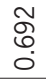 & \& & & & & & & & & & & & & & & & & & & & & & & & \\
\hline e!luy & $\begin{array}{l}\stackrel{1}{\infty} \\
\text { m } \\
0\end{array}$ & 8. & & & & & & & & & & & & & & & & & & & & & & & & \\
\hline peluit & $\begin{array}{l}8 \\
8 \\
\\
0\end{array}$ & & & & & & & & & & & & & & & & & & & & & & & & & \\
\hline גем!!|기 & 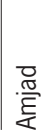 & 旁 & 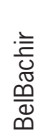 & $\mid \begin{array}{l}:= \\
0 \\
0 \\
0\end{array}$ & 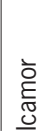 & 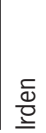 & 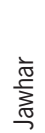 & $\begin{array}{l}\frac{E}{\mathbb{E}} \\
\underline{\underline{T}}\end{array}$ & 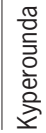 & 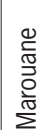 & $\begin{array}{l}\frac{N}{\sqrt[J]{N}} \\
\frac{N}{N} \\
\sum\end{array}$ & $\begin{array}{l}\frac{\pi}{0} \\
\frac{0}{0} \\
\frac{\mathbb{d}}{\Sigma}\end{array}$ & 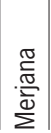 & 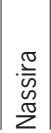 & 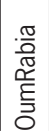 & $\begin{array}{l}\bar{c} \\
\overline{000} \\
\overline{0}\end{array}$ & 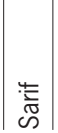 & 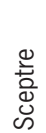 & $\begin{array}{l}\vec{z} \\
\stackrel{0}{0} \\
\infty\end{array}$ & $\left|\begin{array}{l}\frac{\pi}{d} \\
\frac{0}{\mathscr{D}} \\
\mathscr{N}\end{array}\right|$ & 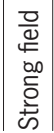 & 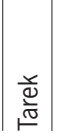 & $\begin{array}{l}\frac{\sqrt{W}}{\omega} \\
\frac{\pi}{\omega}\end{array}$ & 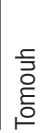 & 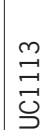 & 홀 \\
\hline
\end{tabular}


NJ method had grouped the durum wheat cultivars into 6 groups at a genetic distance level of 0.25 (Figure 1), and the exotic durum wheat cultivars Sceptre, Medora and Strong field grouped together and formed a single cluster. Selbera, Sebou, Kyperonda formed a separated

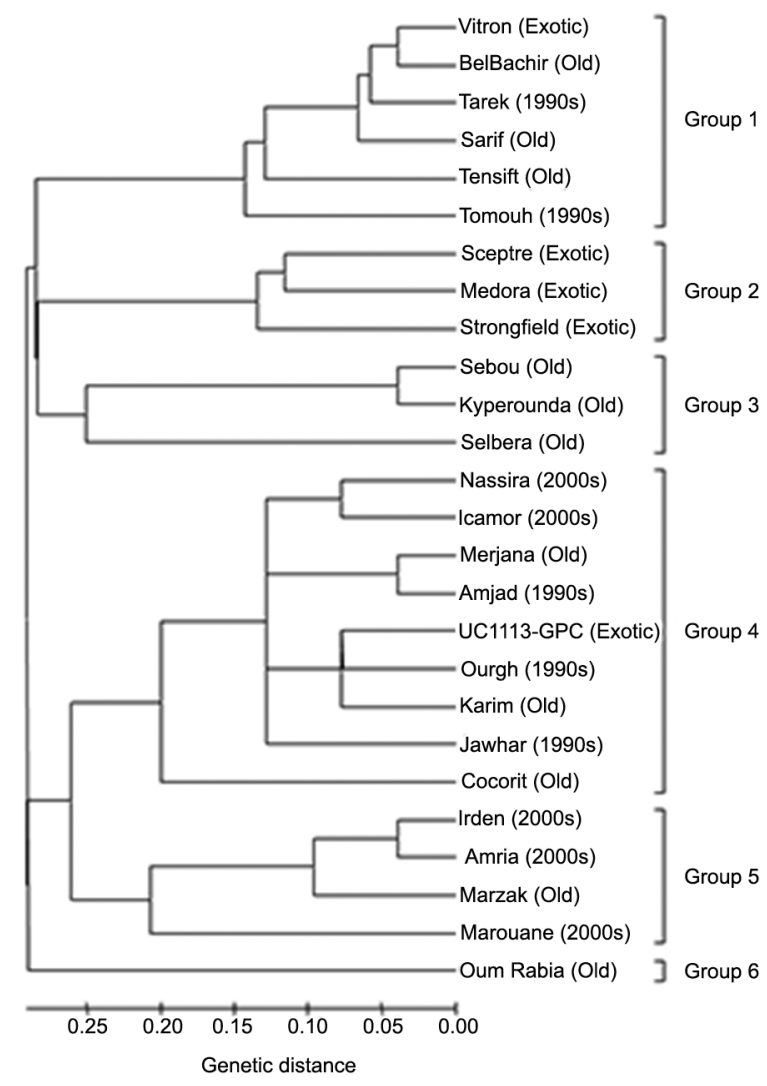

Figure 1 - Dendrogram showing relationships between the 21 Moroccan and 5 exotic cultivars of durum wheat as revealed by the Neighbor-joining method based on shared allele genetic distance. The temporal group or the origin of the cultivar is indicated in parenthesis. cluster. Other two exotic cultivars (Vitron and UC1113Gpc-B1) were embedded in one cluster where the other Moroccan cultivars are grouped into. PCoA analysis (Figure 2) showed similar results similar to the pattern of NJ method clustering and no clear clustering of varieties to any temporal group was observed.

\section{Changes in genetic diversity of durum wheat over time}

To analyze the changes in genetic diversity over periods, the 21 cultivars were grouped into three groups ('old cultivars' released before 1990, the 1990s and the 2000s) according to their year of registration. The total number of alleles and unique alleles were highest in cultivars developed before 1990, decreasing in cultivars developed during the 1990s and increasing slightly in cultivars developed during the 2000s (Table 3). The FPtest clearly showed that decreases in allelic counts in the 1990s and 2000s temporal groups in comparison to the group with 'old cultivars' released before 1990 were significant $(p<0.05$; Table 5). However, the slight increase in allelic counts in the 2000 s temporal group in comparison to the 1990s group was not significant $(p>0.19)$.

Genetic diversity, total alleles and unique alleles were highest in the 'before 1990' temporal group, decreasing in the 1990s, increasing slightly in the 2000's temporal group due to breeding. However, the total genetic diversity of the 1990s and 2000s temporal group was still less than in those cultivars belonging to the 'before 1990' temporal group. AMOVA analysis (Table 6) indicated that most of the molecular variation (91\%) exists among cultivars within temporal groups, with lesser

Table 5 - P-values (based on 50,000 permutations) for FPTest for testing significance of allelic counts between the temporal groups.

\begin{tabular}{lcccc}
\hline & Before 1990 & $1990 \mathrm{~s}$ & $2000 \mathrm{~s}$ & Exotic \\
\hline Before 1990 & - & 0.0033 & 0.0401 & 0.0411 \\
$1990 \mathrm{~s}$ & & - & 0.1934 & 0.1935 \\
$2000 \mathrm{~s}$ & & & - & 0.5524 \\
Exotic & & & & - \\
\hline
\end{tabular}

\section{Principal Coordinates (PCoA)}

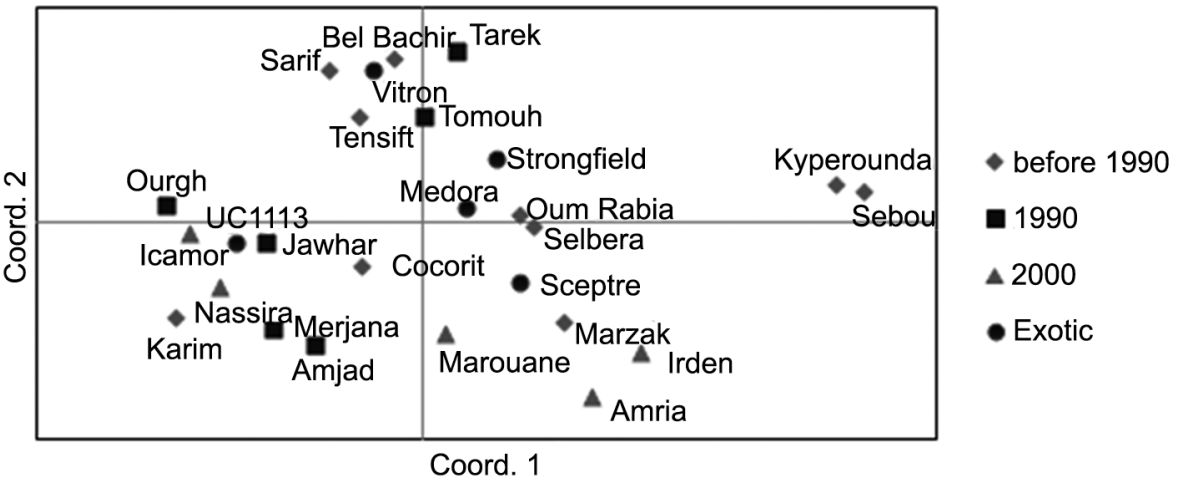

Figure 2 - Principal Coordinate Analysis (PCoA) plot of the Moroccan and exotic durum wheat cultivars based on genetic distance. 
Table 6 - Analysis of molecular variance (AMOVA) of durum wheat cultivars from Morocco and exotic origin based on 13 microsatellite marker analysis.

\begin{tabular}{|c|c|c|c|c|c|}
\hline Source & Degrees of freedom (Df) & Sum of squares (SS) & Mean squares (MS) & Est. Var. & $\%$ \\
\hline Among temporal groups & 3 & 58.872 & 19.624 & 1.180 & $9^{*}$ \\
\hline Within temporal groups & 22 & 268.667 & 12.212 & 12.212 & $91^{*}$ \\
\hline Total & 25 & 327.538 & & 13.392 & 100 \\
\hline
\end{tabular}

*Significant at $p<0.05$.

amounts between temporal groups (9 \%). Permutation tests (based on 999 permutations) suggest that the overall ФРТ was different from the nil distribution $(\Phi P T=$ $0.088, p<0.05)$. Moreover, there was a reduction in genetic variation between the 'before 1990' and 1990s temporal groups $(\Phi \mathrm{PT}=0.01, p<0.05)$. However, the increase in genetic variation between the 1990s and 2000s temporal groups was not significant.

The genetic distances for each pair of temporal groups are summarized in Table 7 . The highest genetic distance (0.431) was found between the 2000s and the exotic groups, whereas the lowest $(0.272)$ was observed between the before 1990 and 1990s temporal groups. The cultivars of exotic origin and the before 1990 temporal group clustered separately, whereas the temporal groups from the 1990s and the 2000s grouped together (Figure 3).

\section{Discussion}

The microsatellite analysis has generated information on cultivar relatedness, which is very useful for the breeding program for identification of suitable cultivars to be used as parents in the crossing programs. A set of 13 microsatellites markers was used for deciphering genetic relationships and the characterization of 21 Moroccan durum wheat cultivars and five exotic cultivars. The microsatellites markers used in this study were sufficient to differentiate all the cultivars and can be used as fingerprints for varietal identification. The marker Xgwm577, Xgwm389 and Xgwm136 were the most informative and showed higher genetic diversity value.

The average number of alleles detected in this study in Moroccan durum wheat cultivars was low (3.38) compared with other recent studies in Tunisia (10.4 alleles; Medini et al., 2005), Syria (7.97 alleles; Achtar et al., 2010), Ethiopia (9.2 alleles; Haile et al., 2013), Iran (5.5 alleles; Mardi et al., 2011) and Italy (4.3 alleles; Figliuolo et al., 2007), indicating a narrow genetic base of Moroccan durum wheat germplasm compared to other countries. Furthermore, our study showed a lower $(p<$ 0.05) number of allelic counts in the 1990s and 2000s temporal groups compared to the before 1990 temporal group. The proportional SSR variations within the improved 1990s and 2000s temporal groups were consistently far lower than those within the older cultivars developed before the 1990s. These findings are clearly in line with observations by $\mathrm{Fu}$ and Somers, (2011) which demonstrate the association between allelic changes and
Table 7 - Genetic distance between the cultivar registers before 1990 (Old cultivars), the 1990s, 2000s and the exotic durum wheat cultivars.

\begin{tabular}{lcccc}
\hline \multirow{2}{*}{ Groups } & \multicolumn{3}{c}{ Genetic distance } \\
\cline { 2 - 5 } & Before 1990 (Old) & 1990s & 2000s & Exotic cultivars \\
\hline Before 1990 (Old) & 0.000 & 0.272 & 0.385 & 0.338 \\
1990s & & 0.000 & 0.295 & 0.308 \\
2000s & & & 0.000 & 0.431 \\
Exotic cultivars & & & & 0.000 \\
\hline
\end{tabular}

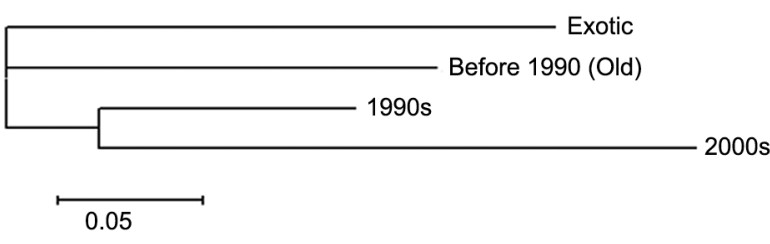

Figure 3 - Dendrogram showing relationships between the three temporal groups and exotic cultivars of durum wheat as revealed by the Neighbor-joining method based on shared allele genetic distance. The scale indicates the genetic distance.

wheat trait improvements, and are useful for understanding the genetic modification of the wheat genome by long-term wheat breeding. The exotic durum lines currently being deployed in breeding programs will enhance the genetic base of the cultivar in Morocco.

Microsatellites were efficient for studying temporal genetic variation. For instance, studies on temporal changes had reported a reduction in genetic diversity in Italian durum wheat, and breeding processes had been attributed to the reduction compared to landraces (Figliuolo et al., 2007) which contradicted the results of Bulgarian durum wheat (Landjeva et al., 2006), and European winter wheat (Huang et al., 2007) where they reported no declining trends in diversity attributable to the breeding process.

The close genetic relationships observed between a number of the cultivars were explained by the presence of common parents in their pedigree. For instance, the close genetic relationship of durum wheat cultivar Vitron with several of the Moroccan cultivars is also obvious, because many of the Moroccan cultivars are either sister lines of Vitron or have Vitron or its sister lines as one of the parents (Nsarellah et al., 2005). This study is the first to report on genetic characterization of the durum wheat cultivars of Morocco. 
In the temporal groups, we detected a decrease in allelic richness in the 1990s and 2000s groups compared with that of the period before 1990 for Moroccan durum wheat cultivars. The slight increase in allelic richness in the 2000s could be explained by the use of varieties introduced and hybridization employing new exotic germoplasm (Jlibene and Nsarellah, 2011). Genetic distance estimates also clearly showed that the durum wheat cultivars of temporal groups before 1990 and the 1990s were closely related compared to genetic distance estimates between the recent temporal group of the 2000s and exotic cultivars, indicating there is an increase in genetic relatedness between the temporal groups which indicates a decrease in genetic diversity. Since there is less of a similarity between temporal groups and exotic cultivars the latter can be employed as parents in a Moroccan breeding program. These findings clearly demonstrate the various natures of the impact of breeding on Moroccan durum wheat cultivars, not only through a reduction in allelic richness but also through a change in genetic relatedness in the released cultivars.

AMOVA showed higher genetic diversity of cultivars within temporal groups $(91 \%)$ compared to that between the temporal groups $(9 \%)$. A reduction in genetic diversity due to breeding occurring since the 1990s was significant. Similar studies have reported that genetic diversity losses have been observed in recent times attributable to breeding in bread wheat /Christiansen et al., 2002; Reif et al., 2005; Warburton et al., 2006; Huang et al., 2007; Hysing et al., 2008). Even though there was an increase in allelic richness in the recent temporal group (the 2000s) compared to the 1990s, attributable to breeding using exotic germplasm from ICARDA/CIMMYT, overall genetic diversity did not increase $(p>$ 0.19). Thus, there is a need to improve further durum wheat productivity and diversity in order to adapt to climate change and emerging pathogens/pests which have been posing real problems in recent years. Exotic durum wheat germplasm are being used as parents in the breeding program for improving productivity and enhancing the genetic diversity of durum wheat on-farm.

\section{Acknowledgements}

The authors are grateful to the International Treaty for Plant Genetic Resources for Food and Agriculture/FAO and the ICARDA/Morocco Collaborative Grants Program for the support. We thank Drs. Anil Kumar and Abhishek Rathod, ICRISAT, India for helping to carry out the FPtest. SMU and FH thank CRP-Wheat for the support.

\section{References}

Achtar, S.; Moualla, M.Y.; Kalhout, A.; Röder, M.S.; Mirali, N. 2010. Assessment of genetic diversity among Syrian durum (Triticum ssp. durum) and bread wheat (Triticum aestivum L.) using SSR Markers. Russian Journal of Genetics 46: 13201326.
Astarini, A.I.; Plummer, A.J.; Lancaster, A.R.; Yan, G. 2004. Fingerprinting of cauliflower cultivars using RAPD markers. Australian Journal of Agricultural Research 55: 112-124.

Christiansen, M.J.; Anderson, S.B.; Ortiz, R. 2002. Diversity changes in an intensively bred wheat germplasm during the 20th century. Molecular Breeding 9: 1-11.

Devos, K.M.; Bryan, G.J.; Collin, A.J.; Stephenson, P.; Gale, M.D. 1995. Application of two microsatellite sequences in wheat storage proteins as molecular markers. Theoretical and Applied Genetics 90: 247-252.

Figliuolo, G.; Mazzee, M.; Greco, I. 2007. Temporal variation of diversity in Italian durum wheat germplasm. Genetic Resources and Crop Evolution 54: 615-626.

Fu, Y.B.; Peterson, G.W.; Richards, K.W.; Somers, D.; DePauw, R.M.; Clarke, J.M. 2005. Allelic reduction and genetic shift in the Canadian hard red spring wheat germplasm released from 1845 to 2004. Theoretical and Applied Genetics 110: 15051516.

Fu, Y.B.; Peterson, G.W.; Yu, J.K.; Gao, L.; Jia, J.; Richards, K.W. 2006. Impact of plant breeding on genetic diversity of the Canadian hard red spring wheat germplasm as revealed by EST-derived SSR markers. Theoretical and Applied Genetics 112: $1239-1247$.

Fu, Y.B.; Somers, D.J. 2009. Genome-wide reduction of genetic diversity in wheat breeding. Crop Science 49: 161-168.

$\mathrm{Fu}$, Y.B.; Somers, D.J. 2011. Allelic changes in bread wheat cultivars were associated with long-term wheat trait improvements. Euphytica 179: 209-225.

$\mathrm{Fu}$, Y.B. 2010. FPTest: a SAS routine for testing allelic counts. Molecular Ecology Resources 10: 389-392.

Haile, J.K.; Hammer, K.; Badebo, A.; Nachit, M.M.; Röder, M.S. 2013. Genetic diversity assessment of Ethiopian tetraploid wheat landraces and improved durum wheat varieties using microsatellites and markers linked with stem rust resistance. Genetic Resources and Crop Evolution 60: 513-527.

Huang, X.Q.; Wolf, M.; Ganal, M.W.; Orford, S.; Koebner, R.M.D.; Röder, M.S. 2007. Did modern plant breeding lead to genetic erosion in European winter wheat varieties? Crop Science 47: 343-349.

Hysing, S.; Sall, T.; Nybom, H.; Liljeroth, E.; Merker, A.; Orford, S.; Koebner, R.M.D. 2008. Temporal diversity changes among 198 Nordic bread wheat landraces and cultivars detected by retrotransposon-based S-SAP analysis. Plant genetic resources characterization and utilization 6: 113-125.

Islam, M.R. 2004. Genetic diversity in irrigated rice. Pakistan Journal of Biological Sciences 2: 226-229.

Jin, L.; Chakraborty, R. 1993. Estimation of genetic distance and coefficient of gene diversity from single-probe multilocus DNA fingerprinting Data. Molecular Biology and Evolution 11: 120127.

Jlibene, M.; Nsarellah, N. 2011. Wheat breeding in Morocco, a historical perspective. p. 425-442. In: Angus, W.J.; Bonjean, A.; Ginkel, M. van, eds. The world wheat book: history of wheat breeding. Lavoisier, Paris, France.

Khanjari, S.; Hammer, K.; Buerkert, A.; Röder, M.S. 2007. Molecular diversity of Omani wheat revealed by microsatellites. I. Tetraploid landraces. Genetic Resources and Crop Evolution 54: 1291-1300. 
Landjeva, S.; Korzun, V.; Ganeva, G. 2006. Evaluation of genetic diversity among Bulgarian winter wheat (Triticum aestivum L.) cultivars during the period 1925-2003 using microsatellites. Genetic Resources and Crop Evolution 53: 1605-1614.

Liu, K.; Muse, S.V. 2005. PowerMarker: An integrated analysis environment for genetic marker analysis. Bioinformatics 21: 2128-2129.

Mardi, M.; Naghavi, M.R.; Pirseyedi, S.M.; Kazemi Alamooti, M.; Rashidi Monfared, S.; Ahkami, A.H.; Omidbakhsh, M.A.; Alavi, N.S.; Salehi Shanjani, P.; Katsiotis, A. 2011. Comparative assessment of SSAP, AFLP and SSR markers for evaluation of genetic diversity of durum wheat (Triticum turgidum L. var. durum). Journal of Agricultural Science and Technology 13: 905-920.

Medini, M.; Hamza, S.; Rebai, A.; Baum, M. 2005. Analysis of genetic diversity in Tunisian durum wheat cultivars and related wild species by SSR and AFLP markers. Genetic Resources and Crop Evolution 52: 21-31.

Nei, M. 1987. Molecular Evolutionary Genetics. Columbia University Press, New York, NY, USA.

Nsarellah, N.; Amri, A.; Nachit, M. 2005. Durum wheat genetic improvement $=$ Amélioration génétique du blé dur. p. 9-55. In: Abbad Andaloussi, F.; Chahbar, A., eds. La création variétale à l'INRA, méthodologie, acquis et perspectives. INRA-Rabat, Morocco (in French).

Peakall, R.; Smouse, P.E. 2012. GenAlEx 6.5: genetic analysis in Excel. Population genetic software for teaching and research-an update. Bioinformatics 28: 2537-2539.

Reif, J.C.; Zhang, P.; Dreisigacher, S.; Warburton, M.L.; Ginkel, M. van; Hoisington, D.; Bohn, M.; Melchinger, A.E. 2005. Wheat genetic diversity trends during domestication and breeding. Theoretical and Applied Genetics 110: 859-864.

Röder, M.S.; Korzun, V.; Gill, B.S.; Wendehake, K.; Pleaschke, J.; Tixier, M.; Leroy, P.; Ganal, M.W. 1998. A microsatellite map of wheat. Genetics 149: 2007-2023.

Roussel, V.; Koenig, J.; Bechert, M.; Balfourier, F. 2004. Molecular diversity in French bread wheat accessions related to temporal trends and breeding programmes. Theoretical and Applied Genetics 108: 920-930.
Roussel, V.; Leisova, L.; Exbrayat, F.; Stehno, Z.; Balfourier, F. 2005. SSR allelic diversity changes in 480 European bread wheat varieties released from 1840 to 2000 . Theoretical and Applied Genetics 111: 162-170.

Saghai-Maroof, M.A.; Soliman, K.M.; Jorgensen, R.A.; Allard, R.W. 1984. Ribosomal DNA spacer length polymorphisms in barley: Mendelian inheritance, chromosomal location, and population dynamics. Proceedings of the National Academy of Sciences of the United States of America 81: 8014-8018.

Saitou, N.; Nei, M. 1987. The neighbor-joining method: a new method for reconstructing phylogenetic trees. Molecular Biology and Evolution 4: 406-425.

Somers, D.J.; Isaac, P.; Edwards, K. 2004. A high-density microsatellite consensus map for bread wheat (Triticum aestivum L.). Theoretical and Applied Genetics 109: 1105-1114.

Tamura, K.; Peterson, D.; Peterson, N.; Stecher, G.; Nei, M.; Kumar, S. 2011. MEGA5: molecular evolutionary genetics analysis using maximum likelihood, evolutionary distance, and maximum parsimony methods. Molecular Biology and Evolution 28: 2731-2739.

Udupa, S.M.; Robertson, L.D.; Weigand, F.; Baum, M.; Kahl, G. 1999. Allelic variation at (TAA) microsatellite Loci in a world collection of chickpea (Cicer arietinum L.) germplasm. Molecular Genetics and Genomics 261: 354-363.

Wang, H.Y.; Wei, Y.M.; Yan, Z.H.; Zheng, Y.L. 2007. EST-SSR DNA polymorphism in durum wheat (Triticum durum L.) collections. Journal of Applied Genetics 48: 35-42.

Warburton, M.L.; Crossa, J.; Franco, J.; Kazi, M.; Trethowan, R.; Rajaram, S.; Pfeiffer, W.; Zhang, P.; Dreisigacker, S.; Ginkel, M. van. 2006. Bringing wild relatives back to the family: Recovering genetic diversity in CIMMYT improved wheat germplasm. Euphytica 149: 289-301.

Ward, R.; Cregan, P.; Song, Q.; Shi, J.; Gill, B.; Singh, S. 2003. 544 BARC SSRs from Rick Ward. Available at: http://wheat. pw.usda.gov/ggpages/whatsnew/2003.shtml [Accessed Oct. 24, 2003]

Wouw, M. van de; Kik, C.; Hintum, T. van; Treuren, R. van; Visser, B. 2009. Genetic erosion in crops: concept, research, results and challenges. Plant Genetic Resources 8: 1-15. 